\title{
An Unusual Case of Gastric Outlet Obstruction After Embolization of Gastroduodenal Artery Pseudoaneurysm
}

\author{
Muhammad Farhan Ashrafa, d ${ }^{\mathrm{O}}$, Aarshi Vipani $^{\mathrm{b}}$, Asra Batool $^{\mathrm{c}}$
}

\begin{abstract}
Gastric outlet obstruction can occur secondary to intrinsic or extrinsic pathology. Historically peptic ulcer disease was the most common cause of gastric outlet obstruction but now malignancy-associated disease process is more common. Gastric outlet obstruction from mucosal ischemia caused by embolization of gastroduodenal artery is unheard of. This is due to the extensive blood supply of the stomach. We present an unusual presentation of gastric outlet obstruction in a patient with recent embolization of pancreatitis-induced pseudoaneurysm of the gastroduodenal artery. The diagnosis was confirmed with esophagogastroduodenoscopy, computed tomography, and upper gastrointestinal series. The case was managed conservatively with a clear liquid diet and proton pump inhibitors. Repeat upper endoscopies at 1 and 6 months after presentation confirmed disease resolution. No guidelines exist on the management of such cases due to the rarity of the disease.
\end{abstract}

Keywords: Gastroenterology; Embolization; Gastroduodenal artery pseudoaneurysm

\section{Introduction}

Gastric outlet obstruction is a clinical syndrome created by any disease process mechanically limiting gastric emptying. It is characterized by postprandial emesis, abdominal pain, weight loss, and early satiety. Most of the time it is caused by mechanical obstruction but motility disorders may also play a role. Both intrinsic and extrinsic pathology at the level of

Manuscript submitted September 7, 2021, accepted September 28, 2021

Published online November 5, 2021

${ }^{a}$ Department of Internal Medicine, Albany Medical Center, Albany, NY, USA ${ }^{\mathrm{b}}$ Cedars Sinai Medical Center, Los Angeles, CA, USA

'Division of Gastroenterology, Albany Medical Center, Albany, New York, USA

${ }^{\mathrm{d} C o r r e s p o n d i n g ~ A u t h o r: ~ M u h a m m a d ~ F a r h a n ~ A s h r a f, ~ D e p a r t m e n t ~ o f ~ I n t e r n a l ~}$ Medicine, Albany Medical Center Hospital, 15 Woodlawn Ave., Albany, NY 12208, USA.Email: fashraf23@gmail.com

doi: https://doi.org/10.14740/jmc3786 the gastric body, pylorus or duodenum can cause gastric outlet obstruction [1]. Historically, peptic ulcer disease was the most common cause of gastric outlet obstruction but with the widespread use of proton pump inhibitor (PPI) and $\mathrm{H}_{2}$ blockers in recent years, cancer-associated gastric outlet obstruction has now become the most common cause [2]. Other causes of gastric outlet obstruction are relatively uncommon and include gastric tuberculosis, annular pancreas, corrosive ingestion, gastric polyps, Crohn's disease, Bouverete syndrome and gastric volvulus. Gastric outlet obstruction from visceral artery aneurysms is rare and a subject of a few case reports [3, 4]. To the best of our knowledge, no cases are found in the literature of gastric outlet obstruction after embolization of visceral artery aneurysms and pseudoaneurysms. Although safe, embolization of visceral artery aneurysms and pseudoaneurysms can result in various post-procedural complications. We report a one-of-a-kind case of gastric outlet obstruction after embolization of large gastroduodenal artery pseudoaneurysm at the origin of the posterior pancreaticoduodenal artery.

\section{Case Report}

\section{Investigations}

A 45-year-old male patient with a past medical history of gastroesophageal reflux disease (GERD), recurrent alcoholinduced pancreatitis and pseudocyst presented with a 1-week history of upper abdominal pain, anorexia, hematemesis and coffee ground emesis. The patient reported that his pain was mild in intensity, was non-radiating and aggravated on vomiting. He reported nausea with coffee ground emesis mixed with food shortly after eating. Four weeks before presentation, the patient was hospitalized for abdominal pain and was found to have a pseudoaneurysm of the gastroduodenal artery at the origin of pancreaticoduodenal artery (Fig. 1). He underwent successful embolization of this pseudoaneurysm with symptom resolution. He denied any non-steroidal anti-inflammatory drugs (NSAIDs) use, recurrent alcohol use, caustic ingestion or uncontrolled GERD. The patient had no known history of Crohn's disease, tuberculosis, gastric polyps, annular pancreas diabetes or gastroparesis.

On admission, the patient had a blood pressure of 105/55 $\mathrm{mm} \mathrm{Hg}$, a temperature of $37^{\circ} \mathrm{C}$, a heart rate of 76 beats per 


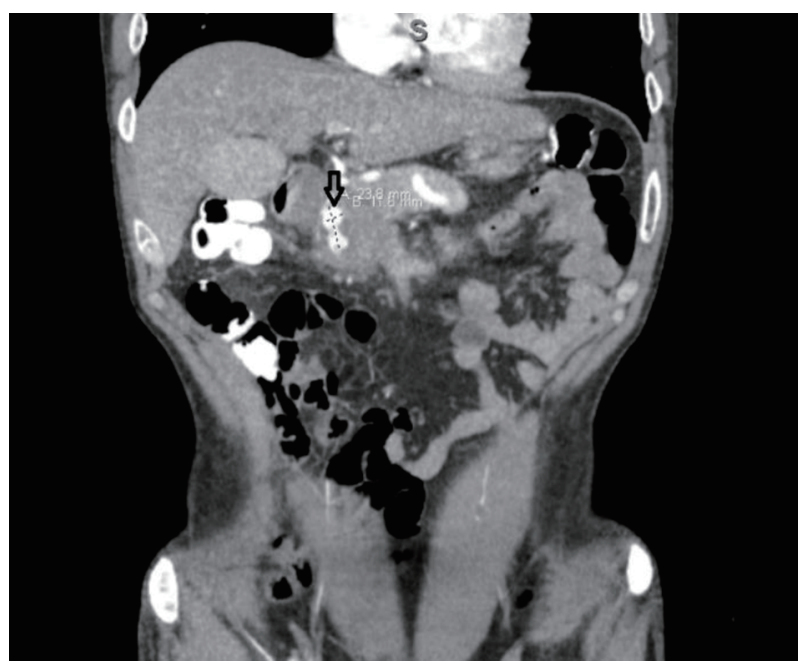

Figure 1. Dumbell-shaped pseudoaneurysm of the gastroduodenal artery before embolization.

minute, and a respiratory rate of 18 cycles per minute. On physical examination, he was in moderate distress while an abdominal examination revealed mild tenderness in the epigastric region. There was no guarding or rebound tenderness.

Laboratory workup showed a white blood count (WBC) of $7,400 / \mu \mathrm{L}$, hemoglobin of $13.3 \mathrm{~g} / \mathrm{dL}$ (down from $15.576 \mathrm{~g}$ / $\mathrm{dL}$ at previous discharge), hematocrit of $40.6 \%$, and a platelet count of $165,000 / \mu \mathrm{L}$.

\section{Diagnosis}

A computed tomography (CT) angiogram of his abdomen was performed to evaluate recent embolization which showed edema along the posterior wall of the stomach and medial wall of the duodenum, adjacent to and surrounding the pancreatic head.

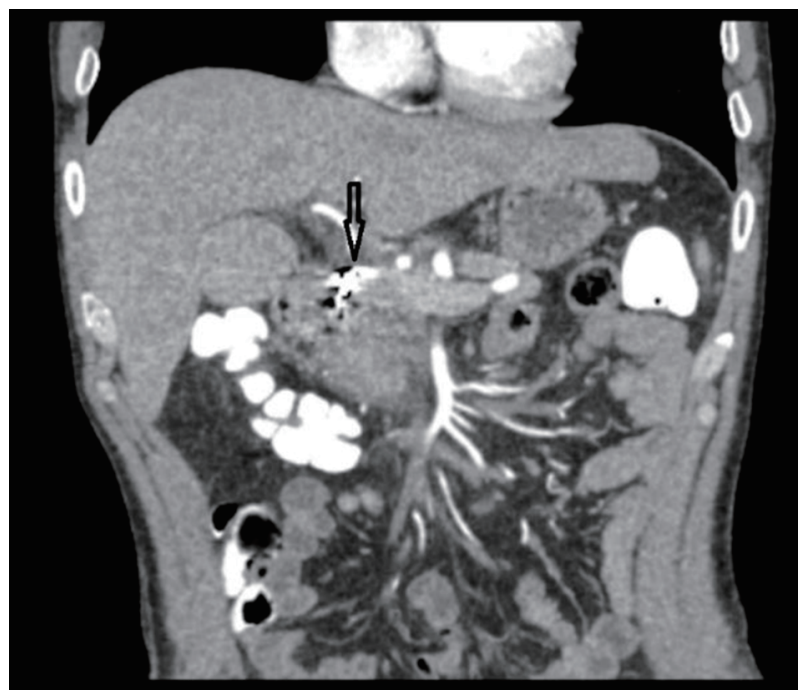

Figure 2. Post-embolization computed tomography scan showing successful occlusion of gastroduodenal artery.

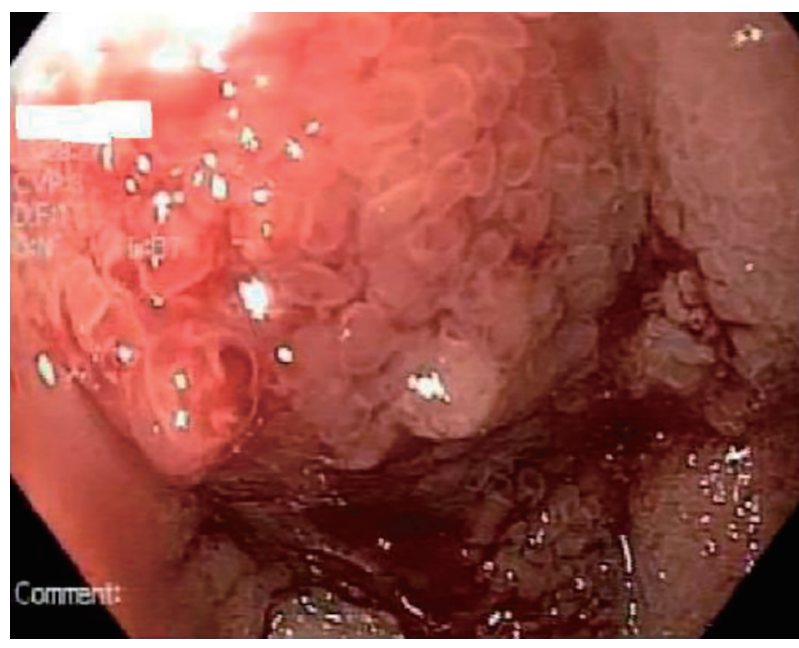

Figure 3. Friable mucosa in gastric antrum and duodenal bulb.

No extrinsic compression of the gastric outlet was seen. No evidence of flow was identified within prior embolized vessels (Fig. 2). Esophagogastroduodenoscopy (EGD) was performed which revealed intense edema, inflammation, and friability in the duodenal bulb causing near obstruction of the gastric outlet (Figs. 3 and 4). Enteric mucosa in the second portion of the duodenum appeared to be normal. There was no apparent bleeding source except the friable mucosa at the duodenal bulb. At this point, differentials including malignancy versus extrinsic compression were considered. Biopsies showed no evidence of malignancy and were negative for Helicobacter pylori. The upper gastrointestinal (GI) series showed compression of the gastric antrum, without evidence of obstruction. A diagnosis of gastric outlet obstruction secondary to ischemia in the setting of recent embolization of gastroduodenal artery was made in the absence of other etiologies.

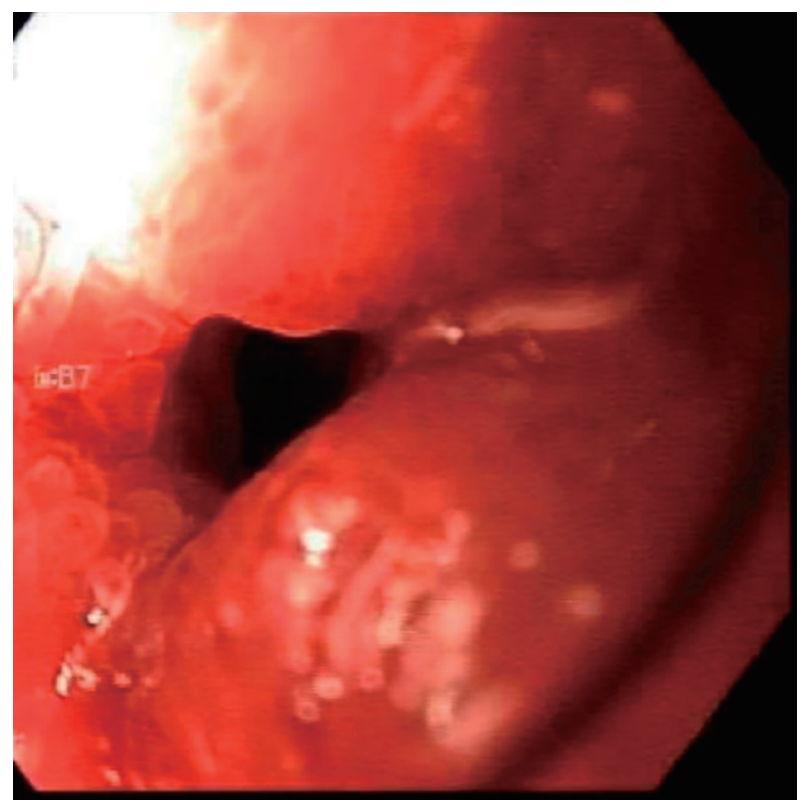

Figure 4. Near obstruction of the first part of duodenum. 


\section{Treatment}

The patient was managed conservatively with a clear liquid diet and pantoprazole $40 \mathrm{mg}$ twice daily. Dilation of gastric outlet obstruction was not attempted due to the friability of mucosa. The plan was made to follow the patient closely with a repeat EGD in a month.

\section{Follow-up and outcomes}

The patient tolerated the liquid diet without any issues. His coffee ground emesis stopped after the initiation of a PPI. A follow-up EGD 1 month later showed some improvement in the stenosed area and a repeat EGD 6 months later showed marked improvement of the obstruction. The patient also improved clinically and was able to advance his diet gradually to a normal diet. No adverse or unanticipated events were noted.

\section{Discussion}

Visceral artery pseudoaneurysms are most commonly found in people 50 - 58 years of age [5]. The male/female ratio is $4.5: 1$ and the mean size is $3.6 \mathrm{~cm}[5,6]$. The development of a pseudoaneurysm in patients with pancreatitis is likely due to periarterial inflammation associated with pancreatitis $[7,8]$. Pancreatitis-induced pseudoaneurysms could affect the splenic $(40 \%)$, gastroduodenal $(30 \%)$, pancreaticoduodenal $(20 \%)$, gastric (5\%), hepatic (2\%), and other (superior mesenteric, jejunal, ileocecal, and aorta) arteries [9, 10]. The most lifethreatening complications of pseudoaneurysms are rupture and subsequent hemorrhage. A pseudoaneurysm tends to enlarge when subjected to sufficient intracystic pressure, ultimately rupturing into the surrounding structures as pseudocyst, GI tract, biliopancreatic ducts, peritoneal cavity, or retroperitoneum. However, the size of the pseudoaneurysm is not a determinant of rupture [11]. Endovascular therapy, including embolization or stent-graft deployment is considered the treatment of choice for hemodynamically stable patients with pancreatitisinduced visceral artery pseudoaneurysms [12]. Complications of endovascular therapy for pseudoaneurysms are rare and include arterial occlusion resulting in bowel infarction, arterial dissection, recurrent bleeding, and infection [13]. However, gastric outlet obstruction is not a common complication.

The presentation of gastric outlet obstruction is non-specific and can mimic patients with malignancy [14]. Patients often present with nausea, vomiting, early satiety, epigastric fullness, pain, anorexia, and some intolerance to solid food. In a later stage, patients may develop significant weight loss due to poor caloric intake. The underlying cause of gastric outlet obstruction is evaluated with plain abdominal radiography, contrast upper GI studies, and EGD [14].

In our case, the upper GI series showed possible compression of gastric antrum with no obstruction to liquids while the CT scan showed no extrinsic compression. An EGD finally confirmed the diagnosis of gastric outlet obstruction by visualization of near-total obstruction of the gastric outlet including duodenal bulb with intense edema. Gastric ischemia is a rare entity due to the abundant stomach blood supply. However, it could occur due to gastric volvulus, hypotension or vascular occlusion, as in our case. Presentation is usually with abdominal angina, diarrhea or gastric bleeding. Gastric mucosal ischemia causing gastric outlet obstruction in absence of peptic ulcer disease is unheard of.

The particularity of this case is the fact that the patient had a pseudoaneurysm at the bifurcation of two visceral arteries and gastric outlet obstruction developed only after the aneurysm was embolized.

\section{Learning points}

This case highlights that while several intra- and post-procedural complications have been reported with endovascular therapy for visceral artery pseudoaneurysms, gastric outlet obstruction is an extremely uncommon finding. It is important to keep this complication in the differential diagnosis in patients with a recent history of endovascular therapy and presenting with gastric outlet obstruction. In addition to its rarity, our case sheds light on the successful management of this rare disorder which may help clinicians in the future.

\section{Acknowledgments}

None to declare.

\section{Financial Disclosure}

There was no financial support provided by any institution or industry.

\section{Conflict of Interest}

There is no conflict of interest to be reported in this case report by any of the authors.

\section{Informed Consent}

Informed patient consent was obtained for publication of the case.

\section{Author Contributions}

MFA served as the primary author of this case report and worked on all the elements right from the introduction to the conclusion. AV worked on discussion, literature review and made sure the chronology of how the case progressed during the course of hospitalization was accurate. AB provided oversight in the case and contributed to the discussion. All authors of this case report had important contributions that led to its final form. 


\section{Data Availability}

Any inquiries regarding supporting data availability of this study should be directed to the corresponding author.

\section{References}

1. Tringali A, Giannetti A, Adler DG. Endoscopic management of gastric outlet obstruction disease. Ann Gastroenterol. 2019;32(4):330-337.

2. Shone DN, Nikoomanesh P, Smith-Meek MM, Bender JS. Malignancy is the most common cause of gastric outlet obstruction in the era of $\mathrm{H} 2$ blockers. Am J Gastroenterol. 1995;90(10):1769-1770.

3. Alhasan A, Khan PM. An unusual cause of gastric outlet obstruction: a pancreaticoduodenal artery aneurysm. Ann Saudi Med. 2009;29(5):393-396.

4. Sharma S, Prasad R, Gupta A, Dwivedi P, Mohindra S, Yadav RR. Aneurysms of pancreaticoduodenal arcade: Clinical profile and endovascular strategies. JGH Open. 2020;4(5):923-928.

5. Matsuzaki Y, Inoue T, Kuwajima K, Ito Y, Okauchi Y, Kondo H, Horiuchi N, et al. Aneurysm of the gastroduodenal artery. Intern Med. 1998;37(11):930-933.

6. Morita Y, Kawamura N, Saito H, Shinohara M, Irie G, Okushiba S, Kato H, et al. [Diagnosis and embolotherapy of aneurysm of the gastroduodenal artery]. Rinsho Hoshasen. 1988;33(5):555-561.
7. Eckhauser FE, Stanley JC, Zelenock GB, Borlaza GS, Freier DT, Lindenauer SM. Gastroduodenal and pancreaticoduodenal artery aneurysms: a complication of pancreatitis causing spontaneous gastrointestinal hemorrhage. Surgery. 1980;88(3):335-344.

8. Quandalle P, Chambon JP, Marache P, Saudemont A, Maes B. Pancreaticoduodenal artery aneurysms associated with celiac axis stenosis: report of two cases and review of the literature. Ann Vasc Surg. 1990;4(6):540-545.

9. Deterling RA, Jr. Aneurysm of the visceral arteries. J Cardiovasc Surg (Torino). 1971;12(4):309-322.

10. Boudghene F, L'Hermine C, Bigot JM. Arterial complications of pancreatitis: diagnostic and therapeutic aspects in 104 cases. J Vasc Interv Radiol. 1993;4(4):551-558.

11. Tessier DJ, Stone WM, Fowl RJ, Abbas MA, Andrews JC, Bower TC, Gloviczki P. Clinical features and management of splenic artery pseudoaneurysm: case series and cumulative review of literature. J Vasc Surg. 2003;38(5):969-974.

12. Coll DP, Ierardi R, Kerstein MD, Yost S, Wilson A, Matsumoto T. Aneurysms of the pancreaticoduodenal arteries: a change in management. Ann Vasc Surg. 1998;12(3):286291.

13. Carr JA, Cho JS, Shepard AD, Nypaver TJ, Reddy DJ. Visceral pseudoaneurysms due to pancreatic pseudocysts: rare but lethal complications of pancreatitis. J Vasc Surg. 2000;32(4):722-730.

14. Khullar SK, DiSario JA. Gastric outlet obstruction. Gastrointest Endosc Clin N Am. 1996;6(3):585-603. 